\title{
Medial Versus Conventional Approach for Prevention of Lateral Cutaneous Nerve Injury in Total hip Arthroplasty with Direct Anterior Approach: A Study for Dual-center, Single-blind, Randomized Controlled Trial
}

\section{Hiroki Tanabe \\ Juntendo Daigaku \\ Yu Ozaki \\ Naotake Yanagisawa \\ Juntendo Daigaku \\ Sammy Bannno \\ Juntendo Daigaku \\ Taiji Watari \\ Juntendo Daigaku \\ Yasuhiro Homma \\ Juntendo Daigaku \\ Kazuo Kaneko \\ Juntendo Daigaku}

Tomonori Baba ( $\nabla$ tobaba@juntendo.ac.jp )

Juntendo Daigaku https://orcid.org/0000-0002-9473-6224

Juntendo Tokyo Koto Koreisha Iryo Center

\section{Study protocol}

Keywords: total hip arthroplasty, direct anterior approach, lateral femoral cutaneous nerve, ultrasonography

Posted Date: August 5th, 2020

DOI: https://doi.org/10.21203/rs.3.rs-31541/v1

License: (c) (i) This work is licensed under a Creative Commons Attribution 4.0 International License. Read Full License 


\section{Abstract}

Background; Lateral cutaneous nerve (LFCN) injury is one of the complication specific to total hip arthroplasty via the direct anterior approach. Recent anatomical studies have revealed that the LFCN not only courses between the sartorius and tensor fasciae latae muscles, but it also branches radially while distributing in the transverse direction from the sartorius muscle to the tensor fasciae latae muscle. The latter is called the fan type, in which injury of the LFCN is almost unavoidable using the conventional site for incision of the fascia. In order to reduce LFCN injury in the fan type, we devised a new approach of incising the fascia at a side $2 \mathrm{~cm}$ medial from the conventional site of incision.

The purpose of this study is to examine how the site of incision of the fascia influences LFCN injury.

Methods; This is a prospective, randomized, controlled study. All patients are divided into the fan type and non-fan type using ultrasonography before surgery. Patients with the fan type LFCN will be performed by the conventional approach and the medial approach in the order specified in the allocation table created in advance by our clinical trial center. The conventional approach involves incision of the skin, followed by incision of the fascia just below the site of skin incision. The medial approach proceeds medially from the site of skin incision, and involves incision of the fascia at a site $2 \mathrm{~cm}$ medial from the conventional site of incision. The primary endpoint is the presence of LFCN injury. The secondary endpoints will be assessed based on PROs at 3 months after surgery in an outpatient setting using the Western Ontario and McMaster Universities Osteoarthritis Index (WOMAC), the Japanese Orthopaedic Association HipDisease Evaluation Questionnaire (JHEQ), and the Forgotten-Joint Score-12 (FJS-12).

Discussion; We hypothesize that the fan type LFCN can be protected from injury by incising the fascia at a more medial site than conventional site. If the usefulness of this surgical procedure is confirmed, the disadvantages of the DAA will be eliminated, potentially being highly beneficial to patients.

Trial registration; UMIN Clinical Trials Registry, UMIN000035944.Registered on 20 Feburary 2019.

\section{Background}

Total hip arthroplasty (THA) is an effective procedure for pain reduction and functional recovery of the hip joint, in addition to QOL improvement $(1,2)$. Among a range of approaches for THA, the direct anterior approach (DAA) has been attracting attention in recent years as a minimally invasive approach because it provides access to the hip joint through intermuscular and internervous planes. As the DAA enables preservation of the soft tissue, the hip joint function can be restored rapidly after THA and a low dislocation rate is achieved. Accordingly, a good postoperative outcome can be expected (3-5). On the other hand, lateral femoral cutaneous nerve (LFCN) injury is one of the complications specific to the DAA (6-9). The LCFN is a sensory nerve in the lateral part of the thigh, and if damaged, symptoms, such as paresthesias and numbness of the skin, may develop. The LFCN is thought to course under the inguinal ligament between the sartorius and tensor fasciae latae muscles $(10,11)$. With the conventional DAA, incision of the fascia is commonly performed at a site slightly lateral from the region between the 
sartorius and tensor fasciae latae muscle in order to avoid LFCN injury. However, the incidence of LFCN injury ranges from $0.1 \%$ to $81 \%$ and it has been reported as a common complication $(1,4,6,10)$. Thus, there may be variations in the course of the nerve apart from those reported previously. Recent anatomical studies have revealed that the LFCN not only courses between the sartorius and tensor fasciae latae muscles, but it also branches radially while distributing in the transverse direction from the sartorius muscle to the tensor fasciae latae muscle (12). The latter is called the fan type, in which injury of the LFCN is almost unavoidable using the conventional site for incision of the fascia (12). Therefore, we hypothesize that the fan type LFCN can be protected from injury by incising the fascia at a more medial site than usual. The purpose of this study is to examine how the site of incision of the fascia influences LFCN injury.

\section{Methods}

\section{Study design}

This is a prospective, dual-center, single-blind, randomized, single-blind, randomized, controlled study.

\section{Ethical aspects and informed consent}

The study was initiated after approval by an ethics committee in January 2019. It is registered with the University Hospital Medical Information Network with an ID of UMIN000035944. All patients who satisfy all of the inclusion criteria and do not meet any of the exclusion criteria will be briefed on this study. After sufficient explanation, consent to participation will be received from the patients themselves.

\section{Study setting}

This study will be conducted in the Juntendo University Hospital and the Juntendo Tokyo Koto Geriatric Medical Center.

\section{Eligibility criteria}

\section{Inclusion criteria}

Prior to enrollment, patients must comply with all of the following:

- Age 20-90 years

- Primary THA via DAA

- Patients who provide written consent for participation after receiving sufficient 
explanation and understanding the study content.

\section{Exclusion criteria}

Patients are excluded if presenting any of the following:

- Trauma

- A history of hip surgery

- Purulent coxitis

- failure to follow instructions of physicians

- Neurological disorder

- BMI of 35 or greater

- Patients deemed unsuitable for inclusion in the study by the principal investigator

\section{Recruitment and randomization}

Those with the fan type LFCN confirmed preoperatively by ultrasonography will be enrolled. The conventional approach and the medial approach will be performed in the order specified in the allocation table created in advance by our clinical trial center.

The trial flow chart is showed in Figure1.

The target number of patients is 40 , and they will be randomly allocated to the conventional incision group (20 hips) and new incision group (20 hips) based on an allocation table using a permuted block randomization schedule with a fixed block size of 4 created prior to the start of the study.

\section{Study timeline and data collection}

After providing consent, study subjects will participate in the study for a total of 4 months, consisting of a 1-month pre-observation period and a 3-month post-observation period. The timeline for the assessment and date collection regarding primary and secondary outcomes is shown in Figure 2.

\section{Ultrasonography of LFCN}

Ultrasonography will be performed by an orthopedic surgeon under general anesthesia before surgery in the operating room. All patients will be positioned supine and scanned on the affected side using the LOGIQ e Expert (GE health- care, Japan) with a 12-MHz linear array transducer. Ultrasonography will be performed following the method reported by Zhu et al (13). The ultrasound transducer is placed in the transverse position and first placed $1-2 \mathrm{~cm}$ distal to the lateral inguinal ligament. Initially, the tensor 
fascia latae muscle and the sartorius are imaged. The LFCN is located in the intermuscular space between the tensor fascia latae and the sartorius. After identifying the LFCN, the probe is moved from the proximal to distal hip joint along the region between the sartorius and tensor fascia latae muscle to confirm the LFCN distribution (Figure3-A). An oval or spindle-shaped high-echoic region (epi- and perineurium) with an inner low-echoic region (nerve bundle) present between the sartorius and tensor fasciae latae muscle corresponds to the LFCN or its branch (13). Following the definition made by Rudin et al. based on a cadaver study, several branches with a similar size present between the muscles are defined as the fan type (Figure3-B). The main root or major branch with surrounding thin branches is defined as the non-fan type (Figure3-C)(12).

\section{Surgical procedures}

THA will be performed using the DAA for all patients. Surgical procedures will be performed by 5 hip joint surgeons with experience performing no less than 50 THA procedures through the $\operatorname{DAA}(9,14)$. THA will be performed under general anesthesia on all patients. Patients will be placed in the supine position and operated on using a traction bed and intraoperative fluoroscope (15). The skin incision will begin $2 \mathrm{~cm}$ lateral and distal to the anterior superior iliac spine, and proceed distally for $10 \mathrm{~cm}$ along a line angling toward the head of the fibula. The surgical field will be ensured and the implant will be placed in the same manner in both groups.

\section{Interventions}

The conventional approach involves incision of the skin, followed by incision of the fascia just below the site of skin incision. The new approach, on the other hand, proceeds medially from the site of skin incision, and involves incision of the fascia at a site $2 \mathrm{~cm}$ medial from the conventional site of incision. Incision of the fascia will be performed to the extent that both approaches can be viewed in the surgical field.

\section{Withdrawal criteria}

If a subject declines to participate in the study or withdraws his/her consent, or if unexpected postoperative complications develop, the subject will be withdrawn from the study.

\section{Primary outcomes}

The primary endpoint is the presence of LFCN injury. The presence of neuropathy will be investigated based on a patient-based questionnaire 3 months after surgery on an outpatient basis. Consistent with 
our previous report, LFCN injury is defined as the presence of the following symptoms over the lateral aspect of the thigh, excluding the surgical scar: numbness, dull sensation, tingling or jolt-like sensation, or pain (10).

\section{Secondary outcomes}

The secondary endpoints will be assessed based on patient-reported outcomes (PROs) at 3 months after surgery in an outpatient setting to examine the extent to which LFCN injury affects the QOL. PROs will be evaluated using the Western Ontario and McMaster Universities Osteoarthritis Index (WOMAC) (pain, stiffness, and function subscales), the Japanese Orthopaedic Association Hip-Disease Evaluation Questionnaire (JHEQ), and the Forgotten-Joint Score-12 (FJS-12).

The WOMAC, first reported by Bellamy and Buchanan in 1986, is used worldwide to evaluate the lower limbs, particularly the hip and knee joints, and has a total score range from 0 (best) to 96 (worst). The JHEQ is a validated self-administered questionnaire evaluating the QOL of Asian-lifestyle patients with hip disease; the total range is from 0 (worst) to 84 (best). The FJS-12, another self-administered questionnaire, is a specific and subjective PRO tool that assesses patients' awareness of their knee or hip joint during activities of daily living. Its final score range is from 0 (worst) to 100 (best) (15).

The highest scores reflecting the best results for the WOMAC are 0 and 96 , and JHEQ are 0 and 84 , respectively. Therefore, the lowest and highest scores for each questionnaire are converted to 0 and 100 , respectively.

\section{Sample size}

According to previous studies, the incidence of LFCN injury due to THA with the DAA is $90 \%$ in fan type patients (16). In total, 40 patients, 20 hips each operated on using the conventional approach and the new approach, are considered necessary assuming that the incidence of adverse events is reduced from $90 \%$ to $50 \%$ by employing the new approach using the chi-square test.

\section{Statistical analysis}

To assess the impact of LFCN injury on patient QOL, we will use Mann-Whitney $U$ tests to compare differences in the PROs between patients reporting neuropraxia versus those with no symptoms, and $\mathrm{p}<$ 0.05 will be regarded as significant. All statistical analyses will be performed using SPSS 22 for Mac (SPSS Inc, Chicago, IL).

\section{Data protection}


When handling information related to the conduct of the study, such information will be managed using a study-specific ID that is not related to the personal information of the study subject, with consideration being given to the protection of confidentiality of the study subject. The principal investigator will store all documents related to the conduct of the study on a computer or at the Department of Orthopaedic Surgery in accordance with the Standard Operating Procedures for the Storage of Samples and Information Pertaining to Medical Research Involving Human Subjects until the day on which 5 years have elapsed from the discontinuation or completion of the study, and will dispose of them while paying attention to personal information.

\section{Study quality assurance and control audits}

The ethics committee of the hospital will review the study implementation status every year to determine whether it is appropriate to continue the study. Any changes to the documents used for the review at the time of application must be approved by the hospital ethics committee in advance. If a serious adverse event develops in the hospital, it should be reported promptly to the hospital director and reviewed by the hospital ethics committee to determine whether it is appropriate to continue the study.

\section{Discussion}

Hip arthroplasty with the DAA was reported by Judet et al. in 1947 using the distal part of the SmithPetersen approach (17-19). The DAA has been attracting attention for rapid recovery of function due to the absence of dissection of muscle tendons. It also has the advantage of being the only approach passing through the internervous plane (the sartorius and tensor fasciae latae muscle are innervated by the femoral nerve and superior gluteal nerve, respectively), and is therefore highly unlikely to cause motor paralysis, unlike other approaches. However, the LFCN, a pure sensory nerve originating from the L2 and L3 nerves, may be damaged by the DAA. Several reports have stated that LFCN injury does not affect hip joint function after THA $(6,20,21)$. Indeed, the presence of LFCN injury did not affect improvement in joint function in our previous study (22). In the same group of patients, however, we demonstrated that LFCN injury reduced the QOL after surgery $(10,22)$. Therefore, we believe that reducing LFCN injury is essential for improving the outcome of THA using the DAA.

Rudin et al. reported that there were three patterns of LFCN distribution (sartorius type, posterior type, and fan type) in an anatomical study (12). They argued that LFCN injury is unavoidable in patients with the fan type LFCN whose nerves radiate to the tensor fasciae latae if the conventional DAA is used (12). We divided LFCN distribution patterns into the fan type and non-fan type (posterior type and sartorius type) using ultrasonography before surgery, and reported that the incidence of LFCN injury was $90 \%$ in patients with the fan type LFCN (16). These clinical findings are consistent with the results of the basic study by Rudin et al. We therefore hypothesize that the incidence of neuropathy can be reduced by incising the fascia at a site medial from the conventional site. If the usefulness of this surgical procedure is confirmed, the disadvantages of the DAA will be eliminated, potentially being highly beneficial to patients. 


\section{Trial status}

We are actively recruiting at the time of submission. Patient recruitment started on June 2019 and the study is scheduled for completion in March 2021. The date of enrolment of the first participant to the trial was 3 June 2019. This protocol is version 1.0, dated 20 Feburary 2019. The study is scheduled for completion in March 2021.

\section{Abbreviations}

THA: Total hip arthroplasty; DAA: Direct anterior approach; LFCN: Lateral femoral cutaneous nerve; BMI: Body mass index; QOL: Quality of life; PROs: patient-reported outcomes; WOMAC: Western Ontario and McMaster Universities Osteoarthritis Index; JHEQ: Japanese Orthopaedic Association Hip-Disease Evaluation Questionnaire; FJS-12: Forgotten-Joint Score-12

\section{Declarations}

\section{Acknowledgements}

The authors would like to thank Tamaki Nara, Yuta Jinnai and Seiya Ishii for their intellectual contributions and support.

\section{Authors' contributions}

HT and TB originated the idea for the study, contributed to its design and developed the intervention protocol. $\mathrm{KK}$ is supervisor of the project. TB, TW, YH, SB and YO are the operator who will conduct this study at the hospital. YJ and NY will perform the statistical analysis. The authors read and approved the final manuscript.

\section{Funding}

No external funding will be used. This study is supported by the Advanced Research Foundation, the Juntendo University Hospital, Tokyo, Japan. There is no role for funding body in the design of the study and collection, analysis, and interpretation of data and in writing the manuscript.

\section{Availability of date and materials}

The datasets generated and analyzed during this study are available from the corresponding author on reasonable request. 


\section{Ethic approval and consent to participate}

This study was approved by the research ethics committee of Juntendo University Hospital on January 2019 (reference number:18-235). We have obtained informed consent from all study participants.

\section{Consent for publication}

The pictuers of LFCN will be used to confirm the LFCN distribution. Therefore, written consent will be obtained using our trial documents from all participants.

\section{Competing interests}

The authors declare that they have no competing interests.

\section{Author details}

Department of Orthopaedic Surgery, Juntendo University, 2-1-1 Hongo, Bunkyo-ku, Tokyo, Japan. Department of Orthopaedic Surgery, Juntendo Tokyo Koto Geriatric Medical Center, 3-3-20 Shinsuna, Koutouku, Tokyo, Japan.

\section{References}

1. Kennon RE, Keggi JM, Wetmore RS, Zatorski LE, Huo MH, Keggi KJ. Total hip arthroplasty through a minimally invasive anterior surgical approach. J Bone Joint Surg Am. 2003;85-A Suppl 4:39-48.

2. Bender B, Nogler M, Hozack WJ. Direct anterior approach for total hip arthroplasty. Orthop Clin North Am. 2009;40(3):321-8.

3. Goebel S, Steinert AF, Schillinger J, Eulert J, Broscheit J, Rudert M, et al. Reduced postoperative pain in total hip arthroplasty after minimal-invasive anterior approach. Int Orthop. 2012;36(3):491-8.

4. Restrepo C, Parvizi J, Pour AE, Hozack WJ. Prospective randomized study of two surgical approaches for total hip arthroplasty. J Arthroplasty. 2010;25(5):671-9 e1.

5. Vajapey SP, Morris J, Lynch D, Spitzer A, Li M, Glassman AH. Nerve Injuries with the Direct Anterior Approach to Total Hip Arthroplasty. JBJS Rev. 2020;8(2):e0109.

6. Goulding K, Beaule PE, Kim PR, Fazekas A. Incidence of lateral femoral cutaneous nerve neuropraxia after anterior approach hip arthroplasty. Clin Orthop Relat Res. 2010;468(9):2397-404.

7. Barton C, Kim PR. Complications of the direct anterior approach for total hip arthroplasty. Orthop Clin North Am. 2009;40(3):371-5.

8. Spaans AJ, van den Hout JA, Bolder SB. High complication rate in the early experience of minimally invasive total hip arthroplasty by the direct anterior approach. Acta Orthop. 2012;83(4):342-6. 
9. De Geest T, Fennema P, Lenaerts G, De Loore G. Adverse effects associated with the direct anterior approach for total hip arthroplasty: a Bayesian meta-analysis. Arch Orthop Trauma Surg. 2015;135(8):1183-92.

10. Homma Y, Baba T, Sano K, Ochi H, Matsumoto M, Kobayashi H, et al. Lateral femoral cutaneous nerve injury with the direct anterior approach for total hip arthroplasty. Int Orthop. 2016;40(8):158793.

11. de Ridder VA, de Lange S, Popta JV. Anatomical variations of the lateral femoral cutaneous nerve and the consequences for surgery. J Orthop Trauma. 1999;13(3):207-11.

12. Rudin D, Manestar M, Ullrich O, Erhardt J, Grob K. The Anatomical Course of the Lateral Femoral Cutaneous Nerve with Special Attention to the Anterior Approach to the Hip Joint. J Bone Joint Surg Am. 2016;98(7):561-7.

13. Zhu J, Zhao Y, Liu F, Huang Y, Shao J, Hu B. Ultrasound of the lateral femoral cutaneous nerve in asymptomatic adults. BMC Musculoskelet Disord. 2012;13:227.

14. Goytia RN, Jones LC, Hungerford MW. Learning curve for the anterior approach total hip arthroplasty. J Surg Orthop Adv. 2012;21(2):78-83.

15. Homma Y, Baba T, Kobayashi H, Desroches A, Ozaki Y, Ochi H, et al. Safety in early experience with a direct anterior approach using fluoroscopic guidance with manual leg control for primary total hip arthroplasty: a consecutive one hundred and twenty case series. Int Orthop. 2016;40(12):2487-94.

16. Ozaki Y, Baba T, Homma Y, Tanabe H, Ochi H, Bannno S, et al. Preoperative ultrasound to identify distribution of the lateral femoral cutaneous nerve in total hip arthroplasty using the direct anterior approach. Sicot j. 2018;4:42.

17. Judet J, Judet R. The use of an artificial femoral head for arthroplasty of the hip joint. J Bone Joint Surg Br. 1950;32-b(2):166 - 73.

18. Judet R, Judet J. Technique and results with the acrylic femoral head prosthesis. J Bone Joint Surg Br. 1952;34-b(2):173 - 80.

19. Smith-Petersen MN. Approach to and exposure of the hip joint for mold arthroplasty. J Bone Joint Surg Am. 1949;31a(1):40-6.

20. Bhargava T, Goytia RN, Jones LC, Hungerford MW. Lateral femoral cutaneous nerve impairment after direct anterior approach for total hip arthroplasty. Orthopedics. 2010;33(7):472.

21. Patton RS, Runner RP, Lyons RJ, Bradbury TL. Clinical Outcomes of Patients With Lateral Femoral Cutaneous Nerve Injury After Direct Anterior Total Hip Arthroplasty. J Arthroplasty. 2018;33(9):291926.e1.

22. Ozaki Y, Homma Y, Baba T, Sano K, Desroches A, Kaneko K. Spontaneous healing of lateral femoral cutaneous nerve injury and improved quality of life after total hip arthroplasty via a direct anterior approach. J Orthop Surg (Hong Kong). 2017;25(1):2309499016684750.

\section{Figures}


Figure 1

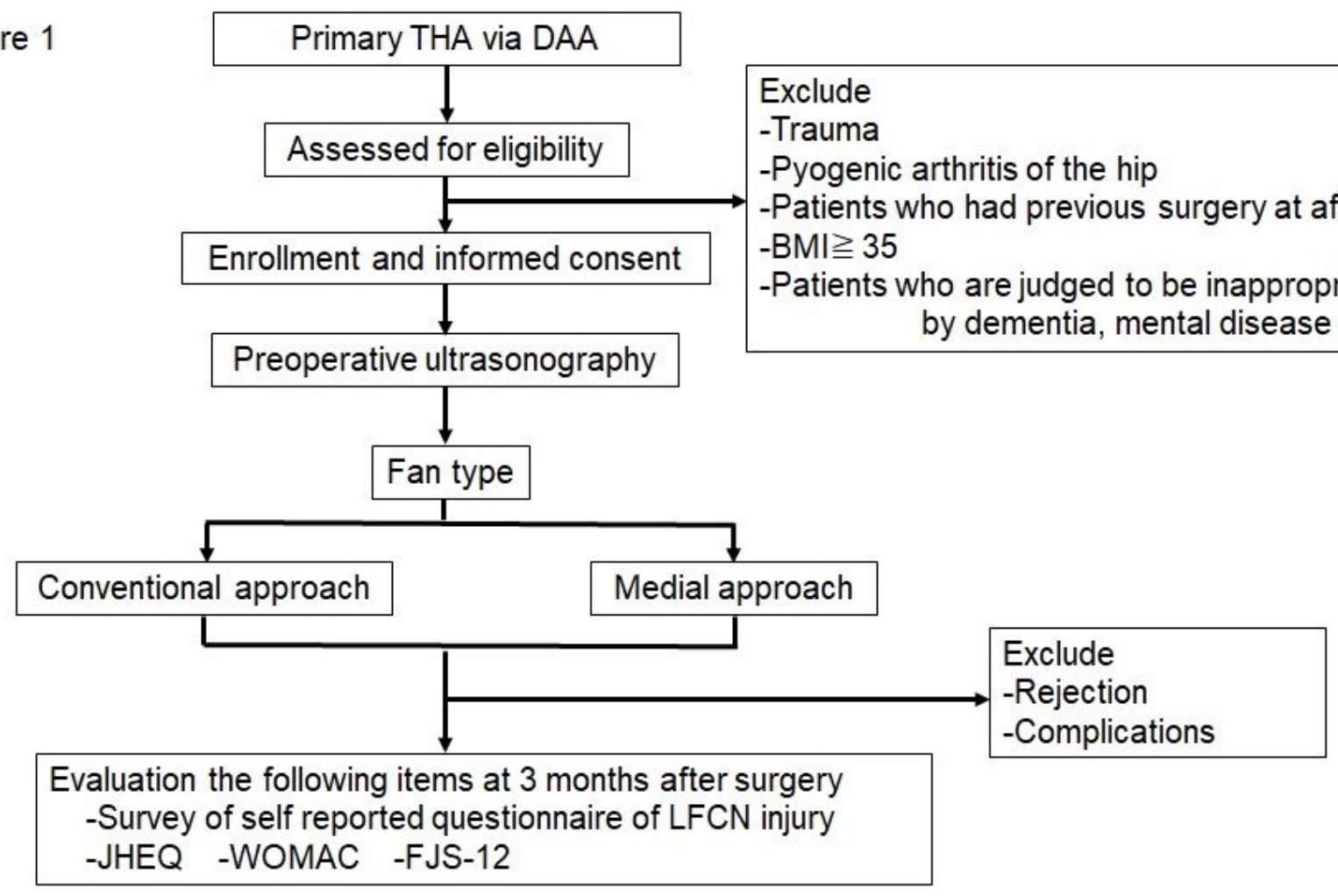

Figure 1

Flow chart of the trial protocol.

Figure 2

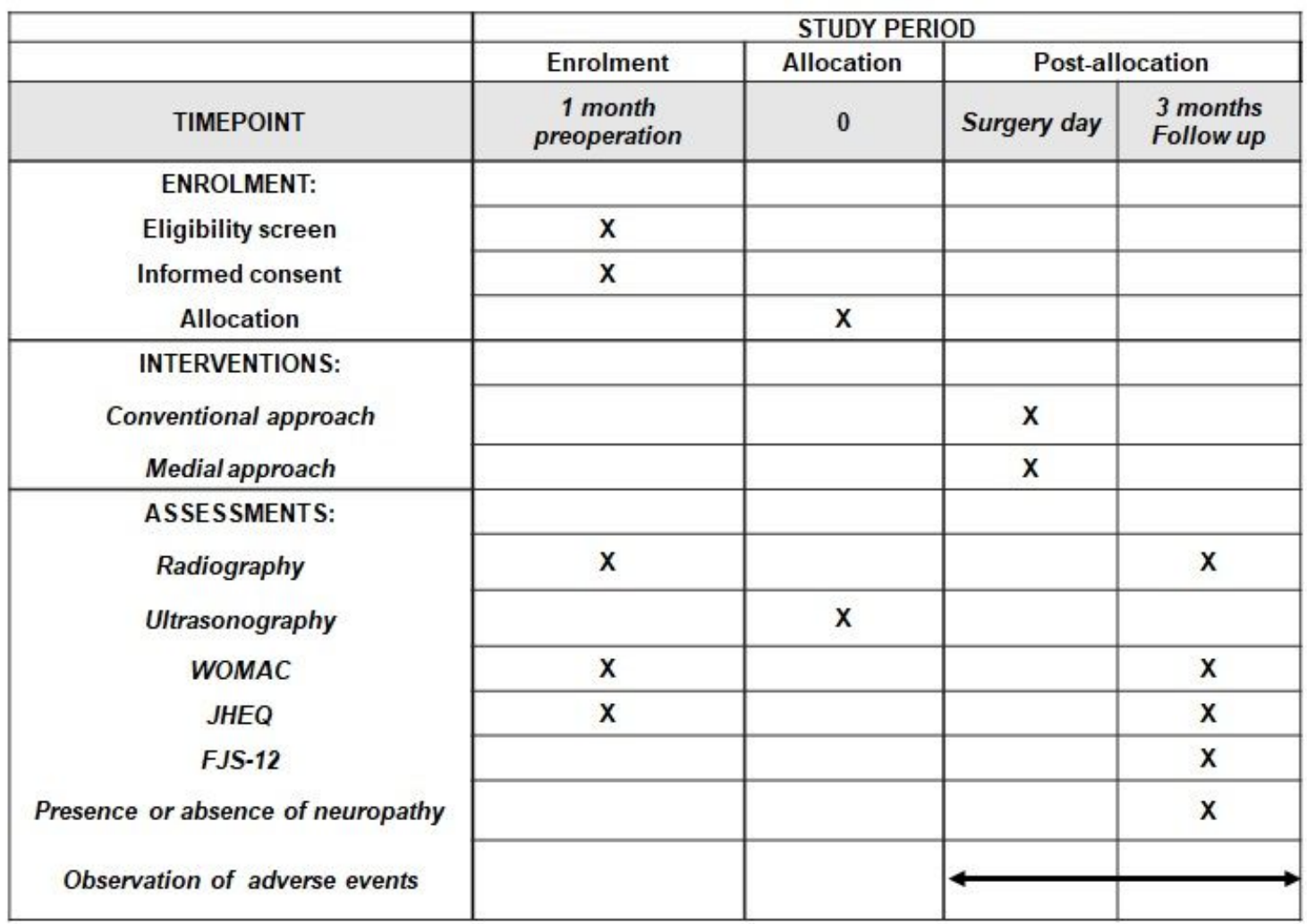


Figure 2

The schedule of enrolment, intervention, and assessments. WOMAC Western Ontario and McMaster Universities Osteoarthritis Index, JHEQ Japanese Orthopaedic Association Hip-Disease Evaluation Questionnaire, FJS-12 Forgotten-Joint Score-12.

Figure 3

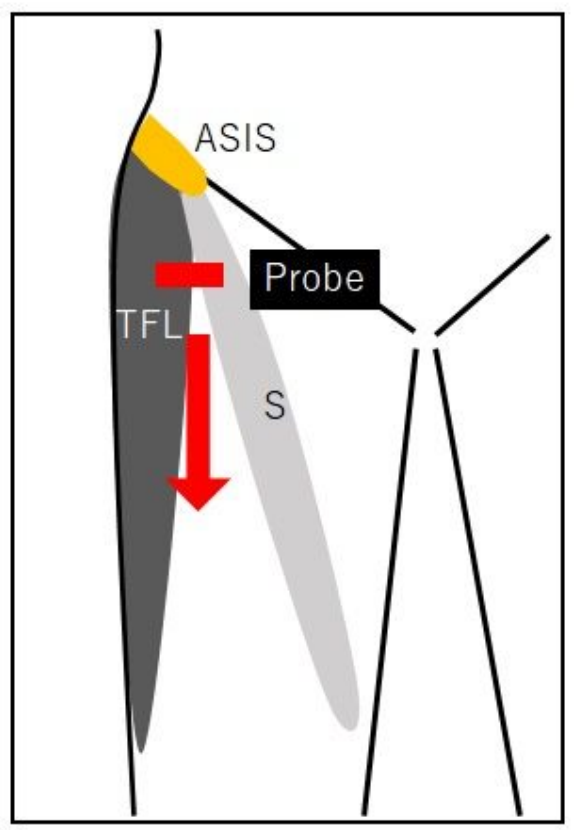

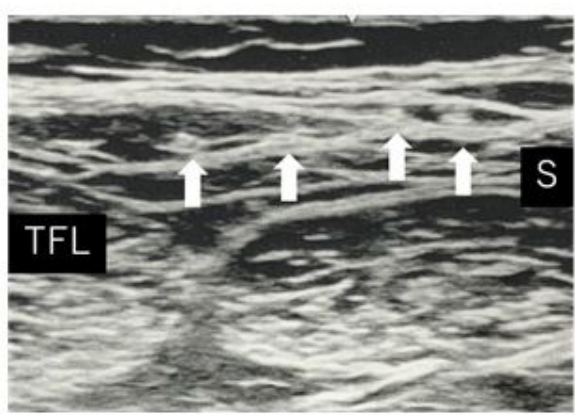

Figure 3-B

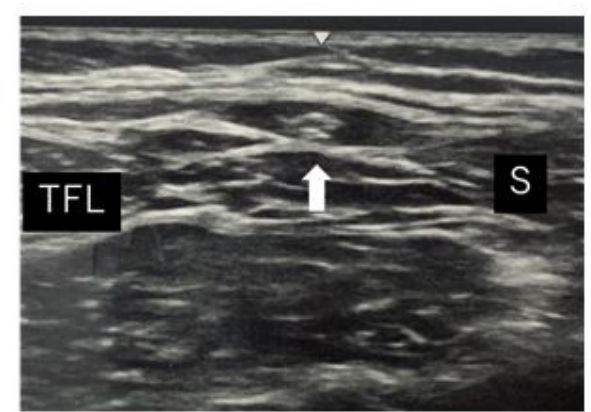

Figure 3-C

Figure 3-A

\section{Figure 3}

A:Shematic diagram showing the initial location of the probe. The probe is moved from the proximal to distal hip joint along the region between the sartorius and tensor fasciae latae muscle (Arrow). B: Transverse ultrasound image of the fan type within the intermuscular space between the sartorius and the tensor fascia latae (Arrows: several nerve branches). C: Transverse ultrasound image of the non-fan type within the intermuscular space between the sartorius and the tensor fascia latae (Arrow: nerve branch). ASIS: Anterior superior iliac spine, TFL: Tensor fascia latae, S: Sartorius

\section{Supplementary Files}

This is a list of supplementary files associated with this preprint. Click to download.

- SPIRITChecklistdownload8Jan13Tanabe.doc 